\title{
Education and Training of Peon on Infection Control: Experience in Peripheral Health Facilities in Nepal
}

\author{
Gagan Gurung \\ Project Manager, Save the Children US, Kathmandu, Nepal
}

doi:10.3396/ijic.V5i1.005.09

\begin{abstract}
Infection control is crucial for the safety of health care workers, patients and individuals in the community who are receiving health care. Peons are employed by the healthcare facilities to support clinical teams, and are responsible for performing tasks which are directly related to blood and body fluids, making them the most vulnerable group of personnel to get infections. Like in most other resource-poor countries they have not been included in any education and training programme concerning Infection Control. In order to address this issue, we designed infection control training programme for peons and assessed their effectiveness to improve Infection Control at peripheral health care facilities in Nepal. A total of 33 health care facilities were randomly selected to participate in this study. It was found that after the training, Infection Control practice increased significantly amongst peons. Environmental cleanliness improved from $24 \%$ to $52 \%$. Similarly, hand washing compliance increased from $36 \%$ to $88 \%$ and waste disposal practices improved from $27 \%$ to $52 \%$. Decontamination of items and equipments increased from $21 \%$ to $45 \%$. Therefore, it can be concluded that education and training of peons, to improve infection control practices in peripheral health care facilities are effective and that support staff can be trained in basic Infection Control using low-tech approaches that are practical and simple thus improving not only their safety but also the safety of patients, individuals and local communities.
\end{abstract}

\section{Key words}

Infection Control, Hand Washing, Waste Disposal, Decontamination, Training, Peons, infection control in low-resource countries, developing countries

\section{Introduction}

Health care-associated infections lead to death, disability and excess medical costs. ${ }^{1}$ One estimation shows that about three million health care workers worldwide are exposed to pathogens such as hepatitis
$B$ virus (HBV), hepatitis $\mathrm{C}$ virus (HCV) and HIV each year, with more than $90 \%$ of these infections occurring in developing countries. ${ }^{2}$ National statistics for 2007 indicate that approximately 70,000 adults and children are infected with HIV virus in Nepal, with an estimated

\section{Corresponding author}

Gagan Gurung, Project Manager, Save the Children US, Kathmandu, Nepal

Email address, gtamu2000@hotmail.com 
prevalence of about $0.49 \%$ in the adult population. ${ }^{3}$ Even though Nepal is experiencing an increase in the burden of communicable diseases, it is difficult to quantify the impact on healthcare associated infection (HCAI) due to the lack of surveillance systems. The risk of $\mathrm{HCAl}$ is even greater in the peripheral government health care facilities (HFs) where health care workers and others often lack the knowledge, skills and resources necessary to carry out even basic infection control practices which is crucial for their safety. In addition, reduction in HCAl maximises patient satisfaction and reduces the cost of health services. ${ }^{4}$

Peripheral health facilities are sub-district level governmental health facilities and included Sub Health Posts (SHP), Health Posts (HP), and Primary Health Care Centres (PHCC). These health facilities provide a range of preventive and curative health care services. The services include immunisation, family planning, antenatal and postnatal care, nutrition education and growth monitoring, health education and treatment of communicable diseases as well as home and health care facilities deliveries. SHP from an institutional perspective is the first contact point for basic health services. Each level above the SHP is a referral point in a network from SHP to HP to PHCC and to district, zonal and finally to specialty tertiary care centres at central level. ${ }^{5}$

As a part of Family Planning and Maternal \& Child Health Project, Save the Children US in collaboration of District Public Health Office (DPHO) conducted joint monitoring of the Infection Control practices of peripheral health facilities of Morang district of Nepal. These monitoring visits showed that the Infection Control practices of most of the HFs were very poor. Paradoxically, the monitoring team found that, a sizable number of health care workers were trained on Infection Control and many of the HFs were equipped with Infection Control materials like puncture proof sharp bins, boiling pots and sterilizers and incinerators. However, use of these resources was found to be quite low. Interestingly, it was observed that peons, the lowest ranking worker in the health services, were conducting tasks and procedures which have both direct and indirect impact on HCAI in many of the HFs.
The major responsibilities of the peons related to Infection Control include waste collection and disposal, cleaning and disinfection of medical items and equipments, environmental cleanliness of HF and its surroundings. In addition to the above responsibilities, these untrained individuals were found to be involved in many other procedures including change of wound dressings, incisions, drainage and suturing of wounds. They were responsible for assisting the majority of procedures directly related to blood and body fluids, making them the most vulnerable group for acquiring as well as transmitting infections. Yet they had rarely been included in any education concerning the maintenance of a healthy environment and in the prevention of cross infection.

In order to address this issue, a Project Team was formed to help design the education and training programme so that effective infection control practices are implemented in these HFs. The programme included 5 days of on-site infection control training to the peons at the selected HFs; followed by 6 to 8 months intensive follow-up programme to assess the impact of training in improving Infection Control at the peripheral health facilities of Nepal.

\section{Methods}

This was a before and after study design. Before the project intervention, an assessment of Infection Control practices were done to assess the extent of the problem. This was followed by 5 days of training to the peons in selected HFs. The training tools used were low-tech approaches that are practical, simple and inexpensive, and thus appropriate to a low-resource setting. ${ }^{4,6}$ The main contents of the training were transmission of microorganisms and infectious disease, hand-washing, appropriate use of personal protective equipment (PPE) e.g. gloving and waste disposal. The methods used in this training included a three-day practical skill based teaching followed by two-day field practices. The training was complemented by intensive follow-up for 6-8 months which focused on practical on-site training. The follow up assessment of the impact of training was done one year after the intervention. A standard infection control observation checklist and guidelines were used to collect data from $\mathrm{HFs}$ for the assessment. The four areas chosen to assess 
the improvement in Infection Control practices were: environmental cleanliness, hand washing practices, waste disposal and decontamination. ${ }^{4,7}$ Out of 66 HFs of Morang district, 33 HFs comprising 3 PHCCs, $7 \mathrm{HPs}$ and 23 SHPs were randomly selected using simple random sampling technique. A complete list of all the HFs was compiled and one by one the HFs were drawn up to the desired sample size. Before collecting data, permission was taken from DPHO, Morang and verbal consent was taken from respondents. Data were entered and analysed using Epi Info 3.2.2 (CDC, Atlanta, USA).

\section{Results}

\section{Environmental cleanliness}

Substantial increase was seen in the cleaning of the health facilities from $28.5 \%$ before training to $72.5 \%$ after training and follow up period (Table I).

\section{Hand washing practices}

Substantial increase was also seen both in hand washing facilities, from $42 \%$ to $82 \%$, and in hand washing compliance which increased form $45 \%$ to 95\% (Table II).

\section{Waste disposal}

Substantial improvement was achieved in medical waste disposal in designated waste containers from $36 \%$ to $94 \%$. However, practices of burning syringes and needles were not quite satisfactory with only $6 \%$ increase after the intervention period.

\section{Decontamination}

Practice of decontamination of instrument with hypochlorite solution before disinfection/sterilization increased from $24 \%$ to $48 \%$ after the training and correct timing for boiling instrument was achieved $21 \%$ to $70 \%$ (Table IV).

\section{Discussion}

This study was focused on measuring the effectiveness of training of peons to improve Infection Control practices in HFs. Four major areas (environment cleanliness, hand-washing facilities and compliance, waste disposal, cleaning and decontamination of instruments) were focused on during training and follow-up periods using 13 indicators. It was found that Infection Control practices improved significantly after education and on-site practical training to the peons.

Table I: Environmental cleanliness

\begin{tabular}{llcr} 
& Indicators & Before & After \\
\hline 1 & Clean floors of HF & $11(33 \%)$ & $25(76 \%)$ \\
\hline 2 & Clean compound of HF & $9(27 \%)$ & $27(82 \%)$ \\
\hline 3 & Clean dressing tables & $10(30 \%)$ & $22(67 \%)$ \\
\hline 4 & Clean toilets & $8(24 \%)$ & $21(64 \%)$ \\
\hline & HF with clean environment (above 1, 2,3,4) & $8(28.5 \%)$ & $17(72.5 \%)$ \\
\hline
\end{tabular}

Table II: Hand washing practices

\begin{tabular}{llcc} 
& Indicators & Before & After \\
\hline 1 & Facilities for hand washing & $14(42 \%)$ & $29(88 \%)$ \\
\hline 2 & Peon washed their hand with soap & $15(45 \%)$ & $31(94 \%)$ \\
\hline
\end{tabular}




\section{Table III: Waste disposal}

\begin{tabular}{llll} 
& Indicators & Before & After \\
\hline 1 & Waste disposed in puncture proofed \& medical waste container & $12(36 \%)$ & $31(94 \%)$ \\
\hline 2 & Pit or incinerator available & $12(36 \%)$ & $24(73 \%)$ \\
\hline 3 & Burning syringe and needles regularly & $18(55 \%)$ & $20(61 \%)$ \\
\hline 4 & Burning combustible items regularly & $19(58 \%)$ & $27(82 \%)$ \\
\hline
\end{tabular}

\section{Table IV: Decontamination}

\begin{tabular}{llll} 
& Indicators & Before & After \\
\hline 1 & Peon has put the instruments in hypochlorite solution & $8(24 \%)$ & $16(48 \%)$ \\
\hline 2 & Peon used utility gloves while cleaning instrument & $7(21 \%)$ & $18(55 \%)$ \\
\hline 3 & Peon has maintained correct timing for boiling instrument & $7(21 \%)$ & $23(70 \%)$ \\
\hline
\end{tabular}

Substantial improvement was achieved in compliance with hand washing practices although improvement in decontamination of instruments was less satisfactory.

An earlier study also conducted in Nepal found similar results with good (94\%) hand washing practices in government health facilities. ${ }^{8}$ The use of gloves $(55 \%)$ and hypochlorite solution (48\%) is still quite low even after the intervention. It might be due to the lack of gloves and hypochlorite solution available in HFs. The practice of decontamination could have further increased but one of the factors which have prevented this was poor supply of materials e.g. hypochlorite solution, gloves and kerosene oil; a problem encountered by most developing countries. A waste disposal and safety measure training to peons conducted by Care-Nepal showed positive outcome with increased waste disposal and safety measure in peripheral HFs of 7 districts of Nepal. ${ }^{9}$

In Nepal, the peon performs many basic and crucial functions related to infection control. Their contribution remains invisible. ${ }^{10}$ This intervention suggested that the training and education program of peons increase their motivation level and they are able to function more efficiently and help improve their safety. It also proved that Infection Control at the peripheral heath facilities, where there is low level of awareness in basic infection control practices and resources are scarce, basic education and training can be improved using simple tools appropriate for support staff. Even with limited staff, equipment and funds, health care facilities in the developing countries can help prevent HCAI and increase safety of healthcare workers, patients and individuals in the community. ${ }^{11}$

It would be unwise to conclude that mere training to these peons would be sufficient to improve infection control of peripheral health facilities. Consideration of other important areas of peripheral health system is necessary to further improve the Infection Control. This study indicates the need for regular supply and availability of materials like kerosene, chlorine solution, soap, water, hand towels and buckets and maintenance of the boilers. The training and education of health care workers on Infection Control is equally important. For Infection Control to be effective, it is essential that HFs have a good and effective infrastructure, managerial support, resources and commitment. Education must include a whole-site training concept for all HCWs, support staff and Health Facility Operation and Management Committee (HFOMC). HFOMC involvement is essential so that they can help address the issues of supply in the local HFs. ${ }^{12}$ 
A limitation of this study is that it used a before-after study design with no comparison groups, therefore it is difficult to establish a cause and effect relationship and it does not take into account temporal change. ${ }^{13}$ Other limitation of this study was the lack of availability of data on needle stick injuries and occupationally acquired infections amongst peons, and the impact on reduction in HCAI during the pre and post intervention periods. This was not possible due to lack of resources and the absence of a surveillance system.

It can be concluded that education and practical training of peons in peripheral HFs where resources are meagre is very effective to improve practices in Infection Control. Therefore it is recommended that peons are offered and included in training on Infection Control, using low-tech approaches that are practical and simple to improve safety for individuals, patients and the community at large.

\section{Acknowledgments}

Author is grateful to DPHO, Morang for providing permission for this work.

\section{Reference}

1. World Health Organization. Infection control, AIDE-MEMOIRE for Infection Prevention and Control in Health Care Facility, 2004.

2. JHPIEGO. Infection Prevention and Control. Available from website - www.jhpiego.org/whatwedo/ipc.htm (Accessed March 13, 2009).

3. Department of Health Services. Department of Health Services, Ministry of Health and Population, Nepal, Annual Report, 2006/2007. Kathmandu: Department of Health Services 2007; 179.

4. Shrestha D. Helping books for Infection prevention. $2^{\text {nd }}$ edn. Nepal: Engender Health 2002; 1-18.

5. Department of Health Services. Department of Health Services, Ministry of Health and Population, Nepal, Annual Report, 2005/2006. Kathmandu: Department of Health Services 2006; 27.

6. Engender Health. Infection Prevention, A Reference Booklet for Health Care Providers, 2001.

7. Disease Prevention and Control Department of Ethiopia. Infection Prevention guidelines for health care facilities in Ethiopia 2004.

8. Subba N, Gurung G. A study of public health indicators of Morang Nepal by lot quality assurance sampling method. Nepal Med Coll J 2007; 9: 117-119.

9. Care Nepal. Project completion report, 2007.

10. Justice J. The invisible worker: the role of the peon in Nepal's health service. Social Science and Medicine 1983; 17: $967-$ 970.

11. Engender Health. Infection Prevention On line Course. Available from website - http://www.engenderhealth.org/ip/ (Accessed March 13, 2009)

12. National Health Training Center, Teku. Directives for local health facility hand over and operation 2003.

13. Thomas S. Before and After Study Designs in Injury Research 2005. Available from website - http://www.pitt.edu/ epi2670/ beforeafter/beforeandafter.ppt (Accessed March 13, 2009) 